\title{
LXXXV. Note on the higher derivative of a function, the variable of which is a function of an independent variable
}

\section{I.J. Schwatt}

To cite this article: I.J. Schwatt (1915) LXXXV. Note on the higher derivative of a function, the variable of which is a function of an independent variable , Philosophical Magazine Series 6, 29:174, 823-831, DOI: 10.1080/14786440608635364

To link to this article: http://dx.doi.org/10.1080/14786440608635364

冓 Published online: 08 Apr 2009.

Submit your article to this journal $[\pi$

Џll Article views: 3

Q View related articles $\sqsubset$ 


\section{[ 823$]$}

LXXXV. Note on the Higher Derivative of a Function, the variable of which is a Function of an independent variable. By I. J. S'chWATT *.

TN E. R. Hedrick's translation of Goursat's work 1 'A Course in Mathematical Analysis' appears the following problem (p. 32, 6.):-

Show that the $n$th derivative of a function $y=\phi(u)$, where $u$ is a function of the independent variable $x$, may be written in the form

(a) $\frac{d^{n} y}{d x^{n}}=A_{1} \phi^{\prime}(u)+\frac{A_{2}}{1.2} \phi^{\prime \prime}(u)+\ldots .+\frac{A_{n}}{1.2 .3 \ldots n} \phi^{n}(u)$,

where

$$
\begin{aligned}
\mathrm{A}_{\kappa}=\frac{d^{n} u^{\kappa}}{d x^{n}}- & \frac{\kappa}{1} u \frac{d^{n} u^{\kappa-1}}{d x^{n}}+\frac{\kappa(\kappa-1)}{1.2} u^{2} \frac{d^{n} u^{\kappa-2}}{d x^{n}}+\ldots \\
& +(-1)^{\kappa-1} \kappa u^{\kappa-1} \frac{d^{n} u}{d x^{n}}(\kappa=1,2, \ldots, n) .
\end{aligned}
$$

[First notice that the $n$th derivative may be written in the form $(a)$, where the coefficients $\mathrm{A}_{1}, \mathrm{~A}_{2}, \ldots, \mathrm{A}_{n}$ are independent of the form of the function $\phi(u)$. To find their values, set $\phi(u)$ equal to $u, u^{2}, \ldots, u^{n}$ successively, and solve the resulting equations for $A_{1}, A_{2}, \ldots, A_{n}$. The result is the form $(b)]$.

I have quoted the problem and the suggestions in full, and shall now proceed to give several proots for it, in the hope that these proofs will illustrate certain operations with series which might be useful in similar work.

I. Let $y=\phi(u)$, wherein $u$ is a function of $x$.

Then

$$
\begin{aligned}
& \frac{d y}{d x}=\frac{d y}{d u} \cdot \frac{d u}{d x}=\phi^{\prime}(u) \frac{d u}{d x} . \\
& \frac{d^{2} y}{d x^{2}}=\phi^{\prime}(u) \frac{d^{2} u}{d x^{2}}+\phi^{\prime \prime}(u)\left(\frac{d u}{d x}\right)^{2} . \\
& \frac{d^{3} y}{d x^{3}}=\phi^{\prime}(u) \frac{d^{3} u}{d x^{3}}+3 \phi^{\prime \prime}(u) \frac{d u}{d x} \frac{d^{2} u}{d x^{2}}+\phi^{\prime \prime \prime}(u)\left(\frac{d u}{d x}\right)^{3} .
\end{aligned}
$$

* Communicated by the Author. 
We now assume

$\frac{d^{n} y}{d x^{n}}=\frac{A_{1}}{1 !} \phi^{\prime}(u)+\frac{A_{2}}{2 !} \phi^{\prime \prime}(u)+\frac{A_{3}}{3 !} \dot{\phi}^{\prime \prime \prime}(u)+\ldots+\frac{A_{n}}{n !} \phi^{n}(u)$,

wherein the $\mathrm{A}^{\prime}$ s are functions of $u$ and $x$, but independent of $\phi(u)$.

To show that (1) is true, we must show that it holds also for the $n+1$ st derivative.

Differentiating (1) with respect to $x$, we have

$\frac{d^{n+1} y}{d x^{n+1}}=\phi^{\prime}(u) \frac{d}{d x} \frac{\mathrm{A}_{1}}{1 !}+\phi^{\prime \prime}(u)\left[\frac{A_{1}}{1 !} \frac{d u}{d x}+\frac{d}{d x} \frac{A_{2}}{2 !}\right]$

$+\phi^{\prime \prime \prime}(u)\left[\frac{\mathrm{A}_{2}}{\overline{2} !} \frac{d u}{d x}+\frac{d}{d x} \frac{\mathrm{A}_{3}}{3 !}\right]+\ldots \phi^{n-1}(u)\left[\frac{\mathrm{A}_{n-2}}{(n-2) !} \frac{d u}{d x}+\frac{d}{d x} \frac{\mathrm{A}_{n-1}}{(n-1)} !\right]$

$+\phi^{n}(u)\left[\frac{\mathrm{A}_{n-1}}{(n-1) !} \frac{d u}{d x}+\frac{d}{d x} \frac{\mathrm{A}_{n}}{n !}\right]+\phi^{n+1}(u) \frac{\mathrm{A}_{n}}{n !} \frac{d u}{d x}$

$=\frac{\mathrm{B}}{1 !} \phi^{\prime}(u)+\frac{\mathrm{B}_{2}}{2 !} \phi^{\prime \prime}(u)+\frac{\mathrm{B}_{3}}{3 !} \phi^{\prime \prime \prime}(u)+\ldots+\frac{\mathrm{B}_{n}}{n !} \phi^{n}(u)+\frac{\mathrm{B}_{n+1}}{(n+1) !} \phi^{n+1}(u)$,

which is of the same form as (1).

We shall now determine the coefficients represented by the A's in (1). Since the A's are independent of $y$, they will have the same value whatever $\phi(u)$ might be.

Letting therefore:

$y=u$, then $\frac{d^{n} u}{d x^{n}}=\mathrm{A}_{1}$;

$y=u^{2}, \quad, \quad \frac{d^{n} u^{2}}{d x^{n}}=2 u \mathrm{~A}_{1}+\mathrm{A}_{2}$;

$y=u^{3}, \quad, \quad \frac{d^{n} u^{3}}{d x^{n}}=3 u^{2} \mathrm{~A}_{1}+3 u \mathrm{~A}_{2}+\mathrm{A}_{3}$

$y=u^{\kappa}, \quad, \quad \frac{d^{n} u^{\kappa}}{d x^{n}}=\left(\begin{array}{c}\kappa \\ 1\end{array}\right) u^{\kappa-1} \mathrm{~A}_{1}+\left(\begin{array}{c}\kappa \\ 2\end{array}\right) u^{\kappa-2} \mathrm{~A}_{2}+\ldots+\left(\begin{array}{c}\kappa \\ \kappa-1\end{array}\right) u \mathrm{~A}_{\kappa-1}+\mathrm{A}_{\kappa}$.

Solving these equations for the A's, we obtain

$$
\begin{aligned}
& \mathbf{A}_{1}=\frac{d^{n} u}{d: x^{n}}, \\
& \mathbf{A}_{2}=\frac{d^{n} u^{2}}{d x^{n}}-\left(\begin{array}{l}
2 \\
1
\end{array}\right) u \frac{d^{n} u}{d x^{n}}, \\
& \mathrm{~A}_{3}=\frac{d^{n} u^{3}}{d x^{n}}-\left(\begin{array}{l}
3 \\
1
\end{array}\right) u \frac{d^{n} u^{2}}{d x^{n}}+\left(\begin{array}{l}
3 \\
2
\end{array}\right) u^{2} \frac{d^{n} u}{d x^{n}} .
\end{aligned}
$$


Higher Derivative of a Function.

Let us now assume

$$
\mathrm{A}_{\kappa}=\sum_{\lambda=0}^{\kappa}(-1)^{\lambda}\left(\begin{array}{c}
\kappa \\
\lambda
\end{array}\right) u^{\lambda} \frac{d^{n}}{d x^{n}} u^{\kappa-\lambda} \ldots . . .
$$

Now if $y=u^{k+1}$, then from (1) follows

$$
\frac{d^{n} u^{\kappa+1}}{d x^{n}}=\left(\begin{array}{c}
\kappa+1 \\
1
\end{array}\right) u^{\kappa} \mathrm{A}_{1}+\left(\begin{array}{c}
\kappa+1 \\
2
\end{array}\right) u^{\kappa-1} \mathrm{~A}_{2}+\ldots+\left(\begin{array}{c}
\kappa+1 \\
\kappa
\end{array}\right) u \mathrm{~A}_{\kappa}+\mathrm{A}_{\kappa+1}
$$

and

$$
\mathrm{A}_{\kappa+1}=\frac{d^{n} u^{\kappa+1}}{d x^{n}}-\sum_{\lambda=1}^{\kappa}\left(\begin{array}{c}
\kappa+1 \\
\lambda
\end{array}\right) u^{\kappa-\lambda+1} \mathrm{~A}_{\lambda} \text {. }
$$

But

$$
\mathrm{A}_{\lambda}=\sum_{\gamma=0}^{\lambda}(-1)^{\gamma}\left(\begin{array}{l}
\lambda \\
\gamma
\end{array}\right) u^{\gamma} \frac{d^{n} u^{\lambda-\gamma}}{d x^{n}},
$$

for values of $\lambda$ from 1 to $\kappa$ inclusive.

Therefore

$$
\mathrm{A}_{\kappa+1}=\frac{d^{n} u^{\kappa+1}}{d x^{n}}-\sum_{\lambda=1}^{\kappa} \sum_{\gamma=0}^{\lambda}(-1)^{\gamma}\left(\begin{array}{c}
\kappa+1 \\
\lambda
\end{array}\right)\left(\begin{array}{l}
\lambda \\
\gamma
\end{array}\right) u^{\kappa+1-\lambda+\gamma} \frac{d^{n} u^{\lambda-\gamma}}{d x^{n}} .
$$

Denoting the double summation by $\mathrm{S}$ and letting $\lambda=\kappa+1-\alpha$, and in turn $\alpha+\gamma=\beta$, we have

$$
\begin{aligned}
\mathrm{S} & =\sum_{\alpha=1}^{\kappa} \sum_{\beta=\alpha}^{\kappa+1}(-1)^{\beta-\alpha}\left(\begin{array}{c}
\kappa+1 \\
\alpha
\end{array}\right)\left(\begin{array}{c}
\kappa+1-\alpha \\
\beta-\alpha
\end{array}\right) u^{\beta} \frac{d^{n} u^{\kappa+1-\beta}}{d x^{n}} \\
& =\sum_{\alpha=1}^{\kappa} \sum_{\beta=\alpha}^{\kappa}(-1)^{\beta-\alpha}\left(\begin{array}{c}
\kappa+1 \\
\alpha
\end{array}\right)\left(\begin{array}{c}
\kappa+1-\alpha \\
\beta-\alpha
\end{array}\right) u^{\beta} \frac{d^{n} u^{\kappa+1-\beta}}{d x^{n}}
\end{aligned}
$$

since for $\beta=\kappa+1, \quad \frac{d^{n} u^{\kappa+1-\beta}}{d x^{n}}=0$.

Now

$$
\sum_{\alpha=1}^{\kappa} \sum_{\beta=\alpha}^{\kappa} \mathbf{M}_{a, \beta}=\sum_{\beta=1}^{\kappa} \sum_{a=1}^{\beta} \mathbf{M}_{a, \beta}
$$

This can be shown in the following way:

$$
\begin{aligned}
\sum_{\alpha=1}^{\kappa} \sum_{\beta=\alpha}^{\kappa} M_{a, \beta}=M_{1,1}+M_{1,2} & +M_{1,3}+\ldots .+M_{1, \kappa} \\
+M_{2,2} & +M_{2,3}+\ldots .+M_{2, \kappa} \\
+ & M_{3,3}+\ldots .+M_{3, \kappa} \\
& +\ldots \ldots \\
& +M_{\kappa, \kappa}
\end{aligned}
$$


Adding by columns we have

$$
\begin{aligned}
\sum_{\alpha=1}^{\kappa} \sum_{\beta=\alpha}^{\kappa} M_{\alpha, \beta} & =M_{1,1}+\left(M_{1,2}+M_{2,2}\right)+\ldots+\left(M_{1, \alpha}+M_{2, \alpha}+\ldots+M_{\kappa, \kappa}\right) \\
& =\sum_{\beta=1}^{\kappa} \sum_{\alpha=1}^{\beta} M_{\alpha, \beta *}
\end{aligned}
$$

By means of (4) the form (3) changes to

$$
\begin{aligned}
\mathrm{S} & =\sum_{\beta=1}^{\kappa}(-1)^{\beta} \sum_{\alpha=1}^{\beta}(-1)^{\alpha}\left(\begin{array}{c}
\kappa+1 \\
\alpha
\end{array}\right)\left(\begin{array}{c}
\kappa+1-\alpha \\
\beta-\alpha
\end{array}\right) u^{\beta} \frac{d^{m} u^{\alpha+1-\beta}}{d x^{23}} \\
& =\sum_{\beta=1}^{\kappa}(-1)^{\beta} u^{\beta} \frac{d^{n} u^{\kappa+1-\beta}}{d x^{n}} \sum_{\alpha=1}^{\beta}(-1)^{\alpha}\left(\begin{array}{c}
\kappa+1 \\
\alpha
\end{array}\right)\left(\begin{array}{c}
\kappa+1-\alpha \\
\beta-\alpha
\end{array}\right) .
\end{aligned}
$$

Now

$$
\begin{aligned}
\left(\begin{array}{c}
\kappa+1 \\
\alpha
\end{array}\right)\left(\begin{array}{c}
\kappa+1-\alpha \\
\beta-\alpha
\end{array}\right) & =\frac{(\kappa+1) !}{(\kappa+1-\alpha) ! \alpha !} \cdot \frac{(\kappa+1-\alpha) !}{(\kappa+1-\beta) !(\beta-\alpha) !} \cdot \frac{\beta !}{\beta !} \\
& =\frac{(\kappa+1) !}{(\kappa+1-\beta) ! \beta !} \cdot \frac{\beta !}{(\alpha-\alpha) ! \alpha !}=\left(\begin{array}{c}
\kappa+1 \\
\beta
\end{array}\right)\left(\begin{array}{c}
\beta \\
\alpha
\end{array}\right) .
\end{aligned}
$$

Therefore

$$
\mathrm{S}=\sum_{\beta=1}^{\kappa}(-1)^{\beta}\left(\begin{array}{c}
\kappa+1 \\
\beta
\end{array}\right) u^{\beta} \frac{d^{n} u^{\alpha+1-\beta}}{d x^{n}} \sum_{\alpha=1}^{\beta}(-1)^{a}\left(\begin{array}{c}
\beta \\
\alpha
\end{array}\right) .
$$

But $\sum_{\alpha=1}^{\beta}(-1)^{a}\left(\begin{array}{l}\beta \\ \alpha\end{array}\right)=\sum_{a=0}^{\beta}(-1)^{a}\left(\begin{array}{l}\beta \\ \alpha\end{array}\right)-1=(1-1)^{\beta}-1=-1$,

and (5) becomes

$$
\mathrm{S}=\sum_{\beta=1}^{\kappa}(-1)^{\beta}\left(\begin{array}{c}
\kappa+1 \\
\beta
\end{array}\right) u^{\beta} \frac{d^{n} u^{\kappa+1-\beta}}{d x^{n}} .
$$

Hence

$$
\begin{aligned}
\mathrm{A}_{\kappa+1} & =\frac{d^{n} u^{\kappa+1}}{d x^{n}}+\sum_{\beta=1}^{\kappa}(-1)^{\beta}\left(\begin{array}{c}
\kappa+1 \\
\beta
\end{array}\right) u^{\beta} \frac{d^{n} u^{\kappa+1-\beta}}{d x^{n}} \\
& =\sum_{\beta=0}^{\kappa}(-1)^{\beta}\left(\begin{array}{c}
\kappa+1 \\
\beta
\end{array}\right) u^{\beta} \frac{d^{n} u^{\kappa+1-\beta}}{d x^{n}}, \text { since } \frac{d^{n} u^{\kappa+1-\kappa-1}}{d x^{n}}=0,
\end{aligned}
$$

and $A_{\kappa+1}$ is of the same form as the one assumed for $A_{\kappa}$ in (2).

II. The following is another method for proving the given theorem. 
Let $\kappa$ successive operations $u \frac{d}{d u}$ each on $\phi(u)$, that is

$$
\left[\left(u \frac{d}{d u}\right)\left(u \frac{d}{d u}\right)\left(u \frac{d}{d u}\right) \ldots \kappa \text { operations }\right] \phi(u)
$$

wherein $u$ and $\frac{d}{d u}$ are not permutable, be designated by

then

$$
\left(u \frac{d}{d u}\right)^{\kappa} \phi(u)
$$

$$
\left(u \frac{d}{d u}\right)^{\kappa} \phi(u)=\sum_{\kappa=1}^{n} \frac{1}{\kappa !} \sum_{a=0}^{\kappa-1}(-1)^{a}\left(\begin{array}{l}
\kappa \\
\alpha
\end{array}\right)(\kappa-\alpha)^{n} u^{\kappa} \frac{d^{\kappa} \phi(u)}{d u^{\kappa}} .
$$

Let $u=e^{x}$, then $\left(u \frac{d}{d u}\right)^{n}=\left(u \frac{\frac{d}{d x}}{\frac{d u}{d x}}\right)^{n}=\left(\frac{d}{d x}\right)^{n}$,
therefore

$$
\frac{d^{n} \phi(u)}{d x^{n}}=\sum_{\kappa=1}^{n} \frac{1}{\kappa !} \sum_{\alpha=0}^{\kappa-1}(-1)^{\alpha}\left(\begin{array}{l}
\kappa \\
\alpha
\end{array}\right)(\kappa-\alpha)^{n} e^{\kappa x} \phi^{(\kappa)}\left(e^{x}\right) .
$$

But

$$
\begin{aligned}
(\kappa-\alpha)^{n} e^{\kappa x} & =e^{\alpha x}(\kappa-\alpha)^{n} e^{(\kappa-\alpha) x} \\
& =e^{\alpha \kappa} \frac{d^{n} e^{(\kappa-\alpha) x}}{d x^{n}} \\
& =u \frac{d^{n} u^{\kappa-\alpha}}{d x^{n}} .
\end{aligned}
$$

Hence

$$
\frac{d^{n} \phi(u)}{d x^{n}}=\sum_{\kappa=1}^{n} \frac{1}{\kappa !} \sum_{\alpha=0}^{\kappa-1}(-1)^{\alpha}\left(\begin{array}{l}
\kappa \\
\alpha
\end{array}\right) u^{\alpha} \frac{d^{n}}{d x^{n}} u^{\kappa-a} \phi^{(\kappa)}(u) .
$$

III. A third way of proving the theorem is as follows :-

$$
\begin{aligned}
& \frac{d y}{d x}=\frac{d y}{d u} \cdot \frac{d u}{d x}, \\
& \frac{d^{2} y}{d x^{2}}=\frac{d^{2} u}{d x^{2}} \cdot \frac{d y}{d u}+\left(\frac{d u}{d x}\right)^{2} \frac{d^{2} y}{d u^{2}},
\end{aligned}
$$

but

$$
\left(\frac{d u}{d . x}\right)^{2}=\frac{1}{2 !}\left[\frac{d^{2} u^{2}}{d x^{2}}-\left(\begin{array}{l}
2 \\
1
\end{array}\right) u \frac{d^{2} u}{d x^{2}}\right]
$$

therefore

$$
\frac{d^{2} y}{d x^{2}}=\sum_{\kappa=1}^{2} \frac{1}{\kappa !} \sum_{\alpha=0}^{\kappa-1}(-1)^{\alpha}\left(\begin{array}{l}
\kappa \\
\alpha
\end{array}\right) u^{\alpha} \frac{d^{2} u^{\kappa-a}}{d x^{2}} \frac{d^{\kappa} y}{d u^{\kappa}} \text {. }
$$


Again $\quad \frac{d^{3} y}{d x^{3}}=\frac{d^{3} u}{d x^{3}} \frac{d y}{d u}+3 \frac{d^{2} u}{d x^{2}} \cdot \frac{d u}{d x} \frac{d^{2} y}{d u^{2}}+\left(\frac{d u}{d x}\right)^{3} \frac{d^{3} y}{d u^{3}}$.

Since

$$
\frac{d^{3} u^{2}}{d x^{3}}=6 \frac{d^{2} u}{d x^{2}} \frac{d u}{d x}+2 u \frac{d^{3} u}{d x^{3}},
$$

and

$$
\frac{d^{3} u^{3}}{d x^{3}}=3 u^{2} \frac{d^{3} u}{d x^{3}}+18 u \frac{d u}{d x} \frac{d^{2} u}{d x^{2}}+6\left(\frac{d u}{d x}\right)^{3},
$$

therefore

$$
3 \frac{d^{2} u}{d x^{2}} \cdot \frac{d u}{d x}=\frac{1}{2 !}\left[\frac{d^{3} u^{2}}{d x^{3}}-\left(\begin{array}{l}
2 \\
1
\end{array}\right) u \frac{d^{3} u}{d x^{3}}\right]
$$

and

$$
\left(\frac{d u}{d x}\right)^{3}=\frac{1}{3 !}\left[\frac{d^{3} u^{3}}{d x^{3}}-\left(\begin{array}{l}
3 \\
1
\end{array}\right) u \frac{d^{3} u^{2}}{d x^{3}}+\left(\begin{array}{l}
3 \\
2
\end{array}\right) u^{2} \frac{d^{3} u}{d x^{3}}\right]
$$

and hence

$$
\begin{aligned}
\frac{d^{3} y}{d x^{3}}= & \frac{d^{3} u}{d x^{3}} \frac{d y}{d u}+\frac{1}{2 !}\left\lceil\frac{d^{3} u^{2}}{d x^{3}}-\left(\begin{array}{l}
2 \\
1
\end{array}\right) u \frac{d^{3} u}{d x^{3}}\right] \frac{d^{2} y}{d u^{2}} \\
& +\frac{1}{3 !}\left\lceil\frac{d^{3} u^{3}}{d x^{3}}-\left(\begin{array}{l}
3 \\
1
\end{array}\right) u \frac{d^{3} u^{2}}{d x^{3}}+\left(\begin{array}{l}
3 \\
2
\end{array}\right) u^{2} \frac{d^{3} u}{d x^{3}}\right] \frac{d^{3} y}{d u^{3}},
\end{aligned}
$$

or written symbolically

$$
\frac{d^{3} y}{d x^{3}}=\sum_{\kappa=1}^{3} \frac{1}{\kappa !} \sum_{\alpha=0}^{\kappa-1}(-1)^{\alpha}\left(\begin{array}{l}
\kappa \\
\alpha
\end{array}\right) u^{\alpha} \frac{d^{3} u^{\kappa-\alpha}}{d x^{3}} \frac{d^{\kappa} y}{d u^{\kappa}} .
$$

Let us assume that this form holds for all values of $n$ from 1 to $n$ inclusive, that is

$$
\frac{d^{n} y}{d x^{n}}=\sum_{\kappa=1}^{n} \frac{1}{\kappa !} \sum_{\alpha=0}^{\kappa-1}(-1)^{\alpha}\left(\begin{array}{c}
\kappa \\
\alpha
\end{array}\right) u^{\alpha} \frac{d^{n} u^{\kappa-\alpha}}{d x^{n}} \frac{d^{\kappa} y}{d u^{\kappa}} \ldots .
$$

We shall then prove that this form holds also for $\frac{d^{n+1} y}{d x^{n+1}}$.

Differentiating (3) gives

$$
\begin{aligned}
\frac{d^{n+1} y}{d x^{n+1}}= & \sum_{\kappa=1}^{n} \frac{1}{\kappa !} \sum_{\alpha=0}^{\kappa-1}(-1)^{\alpha}\left(\begin{array}{l}
\kappa \\
\alpha
\end{array}\right) u^{\alpha} \frac{d^{n_{n} u^{\kappa-\alpha}}}{d x^{n}} \frac{d^{\kappa+1} y}{d u^{\kappa+1}} \frac{d u}{d x} \\
& +\sum_{\kappa=1}^{n} \frac{1}{\kappa !} \sum_{\alpha=0}^{\kappa-1}(-1)^{\alpha}\left(\begin{array}{l}
\kappa \\
\alpha
\end{array}\right) \frac{d}{d x}\left\{u^{\alpha} \frac{d^{n} u^{\kappa-\alpha}}{d x^{n}}\right\} .
\end{aligned}
$$

Now

$$
\sum_{\kappa=1}^{n}\left[\sum_{\alpha=0}^{\kappa-1}(-1)^{\alpha}\left(\begin{array}{l}
\kappa \\
\alpha
\end{array}\right) \frac{d}{d x}\left\{u^{\alpha} \frac{d^{n} u^{\kappa-\alpha}}{d x^{n}}\right\}\right]
$$

$=\sum_{\kappa=2}^{n} \sum_{\alpha=1}^{\kappa-1}(-1)^{\alpha} \alpha u^{\alpha-1} \frac{d u}{d x} \frac{d^{n} u^{\kappa-\alpha}}{d x^{n}}+\sum_{\kappa=1}^{n} \sum_{\alpha=0}^{\kappa-1}(-1)^{\alpha}\left(\begin{array}{c}\kappa \\ \alpha\end{array}\right) u^{\alpha} \frac{d^{n+1} u^{\kappa-\alpha}}{d x^{n+1}}$. 
Therefore

$$
\begin{aligned}
\frac{d^{n+1} y}{d x^{n+1}} & =\sum_{\kappa=2}^{n+1}\left[\sum_{a=0}^{\kappa-1}(-1)^{\alpha}\left(\begin{array}{c}
\kappa-1 \\
\alpha
\end{array}\right) u^{\alpha} \frac{d^{n} u^{\kappa-a-1}}{d x^{n}}\right] \frac{1}{(\kappa-1) !} \frac{d u}{d x} \cdot \frac{d^{\kappa} y}{d u^{\kappa}} \\
& -\sum_{\kappa=2}^{n}\left[\sum_{a=0}^{\kappa-2}(-1)^{\alpha}\left(\begin{array}{c}
\kappa \\
\alpha+1
\end{array}\right)(\alpha+1) u^{\alpha} \frac{d^{n}}{d x^{n}} u^{\kappa-a-1}\right] \frac{1}{\kappa !} \frac{d u}{d x} \frac{d^{\kappa} y}{d x^{\kappa}} \\
& +\sum_{\kappa=1}^{n}\left[\sum_{a=0}^{\kappa-1}(-1)^{\alpha}\left(\begin{array}{c}
\kappa \\
\alpha
\end{array}\right) u^{a} \frac{d^{n+1}}{d x^{n+1}} u^{\kappa-\alpha}\right] \frac{1}{\kappa !} \frac{d^{\kappa} y}{d x^{\kappa}} \ldots . .
\end{aligned}
$$

But

$$
\left(\begin{array}{c}
\kappa \\
\alpha+1
\end{array}\right) \frac{\alpha+1}{\kappa !}=\left(\begin{array}{c}
\kappa-1 \\
\alpha
\end{array}\right) \frac{1}{(\kappa-1) !}
$$

therefore

$$
\begin{aligned}
& \frac{d^{n+1} y}{d x^{n+1}}\left[\sum_{a=0}^{n-1}(-1)^{a}\left(\begin{array}{l}
n \\
\alpha
\end{array}\right) u^{\alpha} \frac{d^{n} u^{n-\alpha}}{d x^{n}}\right] \frac{1}{n !} \frac{d u}{d x} \frac{d^{n+1} y}{d u^{n+1}} \\
& \quad+\sum_{\kappa=1}^{n} \frac{1}{\kappa !} \sum_{a=0}^{\kappa-1}(-1)^{\alpha}\left(\begin{array}{l}
\kappa \\
\alpha
\end{array}\right) u^{a} \frac{d^{n+1} u^{\kappa-\alpha}}{d x^{n+1}} \frac{d^{\kappa} y}{d u^{\kappa}} . .
\end{aligned}
$$

We must now show that

$$
\begin{aligned}
& \frac{1}{n !} \frac{d u}{d x} \frac{d^{n+1} y}{d u^{n+1}}\left[\sum_{\alpha=0}^{n-1}(-1)^{\alpha}\left(\begin{array}{l}
n \\
\alpha
\end{array}\right) u^{\alpha} \frac{d^{n} u^{n-\alpha}}{d x^{n}}-\right] \\
& \quad=\frac{1}{n+1} \frac{d^{n+1}}{d x^{n+1}}\left[\sum_{\alpha=0}^{n}(-1)^{\alpha}\left(\begin{array}{c}
n+1 \\
\alpha
\end{array}\right) u^{\alpha} \frac{d^{n+1} u^{n+1-\alpha}}{d x^{n+1}}\right]
\end{aligned}
$$

i.e.

$$
(n+1) \sum_{\alpha=0}^{n+1}(-1)^{\alpha}\left(\begin{array}{l}
n \\
\alpha
\end{array}\right) u^{\alpha} \frac{d^{n} u^{n-\alpha}}{d x^{n}} \frac{d u}{d x}=\sum_{\alpha=0}^{n}(-1)^{\alpha}\left(\begin{array}{c}
n+1 \\
\alpha
\end{array}\right) u^{\alpha} \frac{d^{n+1} u^{n+1-\alpha}}{d x^{n+1}},
$$

or if we let $n-\alpha=\beta$, we must show that

$$
\begin{aligned}
& (n+1) \sum_{\beta=1}^{n}(-1)^{\beta}\left(\begin{array}{l}
n \\
\beta
\end{array}\right) u^{n-\beta} \frac{d u}{d x} \frac{d^{n} u^{\beta}}{d u^{n}}=\sum_{\beta=0}^{n}(-1)^{\beta}\left(\begin{array}{c}
n+1 \\
\beta+1
\end{array}\right) u^{n-\beta} \frac{d^{n+1} u^{\beta+1}}{d x^{n+1}} \\
& \text { Now } \quad \frac{d^{n+1} u^{\beta+1}}{d x^{n+1}}=(\beta+1) \frac{d^{n}}{d x^{n}}\left(u \frac{d u}{d x}\right)
\end{aligned}
$$

and (6) reduces to

$$
\sum_{\beta=1}^{n}(-1)^{\beta}\left(\begin{array}{l}
n \\
\beta
\end{array}\right) u^{n-\beta} \frac{d u}{d x} \frac{d^{n} u^{\beta}}{d x^{n}}=\sum_{\beta=0}^{n}(-1)^{\beta}\left(\begin{array}{l}
n \\
\beta
\end{array}\right) u^{n-\beta} \frac{d^{n}\left(u^{\beta} \frac{d u}{d x}\right)}{d x^{n}} .
$$



have

By Leibnitz's theorem for the derivative of a product we

$$
\sum_{\beta=0}^{n}(-1)^{\beta}\left(\begin{array}{l}
n \\
\beta
\end{array}\right) u^{n-\beta} \sum_{\gamma=1}^{n}\left(\begin{array}{l}
n \\
\gamma
\end{array}\right) \frac{d^{n-\gamma}}{d x^{n-\gamma}} u^{\beta} \frac{d^{\gamma+1} u}{d x^{\gamma+1}}=0 .
$$

In order to prove (7), it is only necessary to show that it holds for $u=x x^{p}$, when it becomes

$$
x^{p n-n+p-1}\left[\sum_{\beta=0}^{n} \sum_{\gamma=1}^{n}(-1)^{\beta} \frac{(p \beta) !}{\beta ! \gamma !(n-\beta) !(n-\gamma) !(p \beta-n+\gamma) !(p-\gamma+1) !}=0\right.
$$

which is evidently independent of $x$. But when $x$ is a constant, $n$ is also a constant, and therefore (7) holds for all values of $u$. So that:

$$
(n+1) \sum_{\alpha=0}^{n-1}(-1)^{a}\left(\begin{array}{l}
n \\
\alpha
\end{array}\right) u^{a} \frac{d u}{d x} \frac{d^{n} t^{n-\alpha}}{d x^{n}}=\sum_{\alpha=0}^{n}(-1)^{a}\left(\begin{array}{c}
n+1 \\
\alpha
\end{array}\right) u^{\alpha} \frac{d^{n+1}}{d x^{n+1}} u^{n+1-\alpha},
$$

and therefore

$$
\frac{d^{n+1} y}{d x^{n+1}}=\sum_{\kappa=1}^{n+1} \frac{1}{\kappa !} \sum_{\alpha=0}^{\kappa-1}(-1)^{\alpha}\left(\begin{array}{l}
\kappa \\
\alpha
\end{array}\right) u^{a} \frac{d^{n+1} u^{\kappa-\alpha}}{d x^{n+1}} \frac{d^{\kappa} y}{d u^{\kappa}}
$$

The following examples will illustrate the theorem.

To calculate

$$
\text { (i.) } \frac{d^{\kappa}}{d x^{\kappa}} \frac{x^{m}}{\left(1-x^{n}\right)^{p}} \text {. }
$$

By Leibnitz's theorem for the derivative of a product of two functions,

$$
\begin{aligned}
& \frac{d^{\kappa} \frac{x^{m}}{d x^{\kappa}}}{\left(1-x^{n}\right)^{p}}=\sum_{\alpha=0}^{\kappa}\left(\begin{array}{c}
\kappa \\
\alpha
\end{array}\right) \frac{d^{\kappa-\alpha}}{d x^{\kappa-\alpha}} x^{m} \frac{d^{\alpha}}{d x^{\alpha}}\left(1-x^{n}\right)^{-p} \\
& =\kappa ! \sum_{\alpha=0}^{\kappa}\left(\begin{array}{c}
m \\
\kappa-\alpha
\end{array}\right) \frac{x^{m-\kappa+\alpha}}{\alpha !} \frac{d^{\alpha}}{d x^{\alpha}}\left(1-x^{n}\right)^{-p} \\
& =\kappa !\left[\left(\begin{array}{c}
m \\
\kappa
\end{array}\right) \frac{x^{m-\kappa}}{\left(1-x^{n}\right)^{p}}+\sum_{\alpha=1}^{\kappa}\left(\begin{array}{c}
m \\
\kappa-\alpha
\end{array}\right) \frac{x^{m-\kappa+\alpha}}{\alpha !} \frac{d^{\alpha}}{d x^{a}}\left(1-x^{n}\right)^{-p}\right] .
\end{aligned}
$$

Let $x^{n}=u$, and $(1-u)^{-p}=y$, then

$$
\begin{aligned}
\frac{d^{\alpha} y}{d x^{\alpha}} & =\sum_{\beta=1}^{\alpha} \frac{1}{\beta !} \sum_{\gamma=0}^{\beta-1}(-1)^{\gamma}\left(\begin{array}{l}
\beta \\
\gamma
\end{array}\right) x^{n \gamma} \frac{d^{\alpha}}{d x^{\alpha}} x^{n(\beta-\gamma)} \frac{p(p+1)(p+2) . .(p+\beta-1)}{\left(1-x^{n}\right)^{p+\beta}} \\
& =\sum_{\beta=1}^{\alpha} \frac{1}{\beta} \sum_{\gamma=0}^{\beta-1}(-1)^{\gamma}\left(\begin{array}{l}
\beta \\
\gamma
\end{array}\right) \frac{(n \beta-\gamma) !}{(n \bar{\beta}-\gamma-\alpha) !} \frac{(p+\beta-1) !}{(p-1) !} \frac{x^{n \beta-\alpha}}{\left(1-x^{n}\right)^{p+\beta^{*}}}
\end{aligned}
$$


Higher Derivative of a Function.

Therefore

$$
\begin{aligned}
\frac{d^{\kappa}}{d x^{\kappa}} \frac{x^{m}}{\left(1-x^{n}\right)^{p}}=\kappa ! & {\left[\left(\begin{array}{c}
m \\
\kappa
\end{array}\right) \frac{x^{m-\kappa}}{\left(1-x^{n}\right)^{p}}+\sum_{\alpha=1}^{\kappa}\left(\begin{array}{c}
n \iota \\
\kappa-\alpha
\end{array}\right) x^{m-\kappa+a}\right.} \\
& \left.\times \sum_{\beta=1}^{a} \sum_{\gamma=0}^{\beta-1}(-1)^{\gamma}\left(\begin{array}{c}
\beta \\
\gamma
\end{array}\right)\left(\begin{array}{c}
n \beta-\gamma \\
\alpha
\end{array}\right)\left(\begin{array}{c}
p+\beta-1 \\
\beta
\end{array}\right) \frac{x^{n \beta-\alpha}}{\left(1-x^{n}\right)^{p+\beta}}\right] \\
= & \kappa ! \frac{x^{m-\kappa}}{\left(1-x^{n}\right)^{p}}\left[\left(\begin{array}{c}
m \\
\kappa
\end{array}\right)+\sum_{\alpha=1}^{\kappa}\left(\begin{array}{c}
m \\
\kappa-\alpha
\end{array}\right)\right. \\
& \left.\times \sum_{\beta=1}^{\alpha}\left(\begin{array}{c}
p+\beta-1 \\
\beta
\end{array}\right) \frac{x^{m \beta}}{\left(1-x^{n}\right)^{\beta}} \sum_{\gamma=0}^{\beta-1}(-1)^{\gamma}\left(\begin{array}{c}
\beta \\
\gamma
\end{array}\right)\left(\begin{array}{c}
n \bar{\beta}-\bar{\gamma} \\
\alpha
\end{array}\right)\right] .
\end{aligned}
$$

$\underset{\infty}{\infty} \quad$ (ii.) $\quad \frac{d n}{d x^{n}} \frac{1}{\left(1-x^{3}+x^{7}\right)^{p^{2}}}$. Let $x^{3}-x^{7}=u$ and $(1-u)^{-p}=y$, then

$$
\frac{d^{n} y}{d x^{n}}=\sum_{\kappa=1}^{n} \frac{1}{\kappa !} \sum_{a=0}^{\kappa-1}(-1)^{\alpha}\left(\begin{array}{l}
\kappa \\
\alpha
\end{array}\right) u^{\alpha} \frac{d^{n}}{d x^{n}} u^{\kappa-\alpha} \frac{d^{\kappa} y}{d u^{\kappa}}
$$

Now

$$
\frac{d^{\kappa} y}{d u^{\kappa}}=\frac{d^{\kappa}}{d u^{\kappa}}(1-u)^{-p}=\kappa !\left(\begin{array}{c}
p+\kappa-1 \\
\kappa
\end{array}\right)(1-u)^{-(p+\kappa)},
$$

and

\section{Therefore}

$$
\begin{aligned}
\frac{d^{n}}{d x^{n}} u^{\kappa-\alpha} & =\frac{d^{n}}{d x^{n}}\left(x^{3}-x^{7}\right)^{\kappa-\alpha}=\frac{d^{n}}{d x^{n}}\left[x^{3}\left(1-x^{4}\right)\right]^{\kappa-\alpha} \\
& =\frac{d^{n}}{d x^{n}} x^{3(\kappa-\alpha)} \sum_{\beta=0}^{\kappa-\alpha}(-1)^{\beta}\left(\begin{array}{c}
\kappa-\alpha \\
\beta
\end{array}\right) x^{4 \beta} \\
& =n ! \sum_{\beta=0}^{\kappa-\alpha}(-1)^{\beta}\left(\begin{array}{c}
\kappa-\alpha \\
\beta
\end{array}\right)\left(\begin{array}{c}
3 \kappa-3 \alpha+4 \beta \\
n
\end{array}\right) x^{3 \kappa-3 \alpha+1 \beta-1} .
\end{aligned}
$$

$$
\begin{aligned}
& ! \sum_{\kappa=1}^{n} \frac{1}{\kappa} ! \sum_{\alpha=0}^{\kappa-1}(-1)^{a}\left(\begin{array}{l}
\kappa \\
a
\end{array}\right)\left(x^{3}-x^{2}\right)^{a} \\
& \times \sum_{\beta=0}^{\kappa-\alpha}(-1)^{\beta}\left(\begin{array}{c}
\kappa-\alpha \\
\beta
\end{array}\right)\left(\begin{array}{c}
3 \kappa-3 \alpha+4 \beta \\
n
\end{array}\right) x^{3 \kappa-3 \alpha+4 \beta-n} \kappa !\left(\begin{array}{c}
p+\kappa-1 \\
\kappa
\end{array}\right)\left(1-x^{3}+x^{7}\right)^{p-\kappa} \\
& =\frac{n !}{x^{n}\left(1-w^{3}+x^{7}\right)^{p}} \sum_{\kappa=1}^{n} \sum_{\alpha=0}^{\kappa-1}\left(\begin{array}{c}
\kappa \\
\alpha
\end{array}\right)_{\beta=0}^{k-\alpha}(-1)^{\alpha+\beta}\left(\begin{array}{c}
\kappa-\alpha \\
\beta
\end{array}\right) \\
& \times\left(\begin{array}{c}
3 \kappa-3 \alpha+4 \beta \\
n
\end{array}\right)\left(\begin{array}{c}
p+\kappa-1 \\
\kappa
\end{array}\right) \frac{\left(x^{3}-x^{7}\right) x^{3 \kappa-3 a+4 \beta}}{\left(1-x^{3}+x^{7}\right)^{\kappa}} \\
& =\frac{n !}{x^{n}\left(1-x^{3}+x^{7}\right)^{p}} \sum_{\kappa=1}^{n} \sum_{\alpha=0}^{\kappa-1} \sum_{\beta=0}^{\kappa-\alpha}(-1)^{\alpha+\beta}\left(\begin{array}{l}
\kappa \\
\alpha
\end{array}\right)\left(\begin{array}{c}
\kappa-\alpha \\
\beta
\end{array}\right) \\
& \times\left(\begin{array}{c}
3 \kappa-3 \alpha-4 \beta \\
n
\end{array}\right)\left(\begin{array}{c}
p+\kappa-1 \\
\kappa
\end{array}\right) \frac{\left(1-x^{4}\right)^{\alpha} x^{3 \kappa+4 \beta}}{\left(1-v^{3}+\varepsilon^{2}\right)^{\beta}} .
\end{aligned}
$$

University of Pennsylvania,

Philadelphia, Pr., U.S.A. 